\title{
Microarray expression profile of long non-coding RNAs in paclitaxel-resistant human lung adenocarcinoma cells
}

\author{
XIN TIAN $^{1,2}$, HONGYAN ZHANG $^{1}$, BIJUN ZHANG $^{1}$, JIANZHU ZHAO $^{1}$, \\ TINGTING LI ${ }^{1}$ and YANYAN ZHAO ${ }^{1}$ \\ Departments of ${ }^{1}$ Clinical Genetics and ${ }^{2}$ Medical Oncology, Shengjing Hospital of China Medical University, \\ Shenyang, Liaoning 110004, P.R. China
}

Received October 20, 2016; Accepted May 15, 2017

DOI: $10.3892 / o r .2017 .5691$

\begin{abstract}
Paclitaxel (PTX)-based chemotherapy is a standard treatment for human lung adenocarcinoma, but treatment often fails since resistance develops. Recent studies have described the activity of long non-coding RNAs (lncRNAs) in many biological processes and human diseases. Chemotherapy resistance is one of these areas, but the role of lncRNAs in paclitaxel resistance of human lung adenocarcinoma cells has not been reported. A paclitaxel resistance model was established using A549 human lung adenocarcinoma cells. lncRNAs and mRNAs were profiled in parental A549 and paclitaxel-resistant A549/PTX cells by microarray analysis. Real-time quantitative PCR (RT-qPCR) was used to validate the results of the microarray. Chromosomal distribution patterns of differentially expressed lncRNAs and mRNAs were assessed. Gene Ontology (GO) and Kyoto Encyclopedia of Genes and Genomes (KEGG) pathway analyses were performed using gene set enrichment. We screened 1,154 lncRNAs and 1,733 mRNAs that had a >3-fold difference in expression in A549/PTX cells compared with A549 cells, most of which were downregulated. Nine lncRNAs and six mRNAs were randomly selected and validated by RT-qPCR. Most aberrantly expressed lncRNAs and mRNAs were located on chromosomes 1, 2, 6, 12 and 17, particularly on chromosome 1 . Bioinformatics, GO and KEGG pathway analyses, revealed that some differentially expressed genes regulated classical functions and pathways such as cytosol components, protein binding, gene expression and metabolic pathways. Differential expression of lncRNAs and mRNAs in A549/PTX and A549 cells indicates that various lncRNAs may be useful diagnostic or prognostic markers of resistance to treatment, or future targets for paclitaxel-based chemotherapy, providing a novel rationale for clinical treatment.
\end{abstract}

Correspondence to: Professor Yanyan Zhao, Department of Clinical Genetics, Shengjing Hospital of China Medical University, 36 San Hao Street, Shenyang, Liaoning 110004, P.R. China E-mail:yyzhao@sj-hospital.org; yyzhao@mail.cmu.edu.cn

Key words: long non-coding RNA, paclitaxel-resistant, chemotherapy, lung adenocarcinoma, microarray expression profile

\section{Introduction}

Lung cancer is one of the most common human cancers, and with the highest worldwide incidence and mortality (1). Adenocarcinoma is one of the more common pathological types (2). Despite advances in surgery, radiotherapy and targeted treatment, survival is far from satisfactory. Most patients are given paclitaxel (PTX)-based combination chemotherapy as a standard treatment (3). Paclitaxel is a taxane, and its cytotoxic effect depends on microtubule polymerization, and inhibition of microtubule depolymerization leads to a cell cycle block in the $\mathrm{G} 2 / \mathrm{M}$ phase resulting in tumor cell apoptosis or necrosis (4). However, eventual development of resistance to paclitaxel is inevitable and leads to treatment failure. The paclitaxel mechanism of resistance is multifactorial and complex, involving changes in drug-efflux, increased drug metabolism, interference with DNA repair and cell cycle regulation, and disorders of cell apoptosis and autophagy $(5,6)$. Despite our knowledge of its anticancer effects, little is known concerning the development of paclitaxel resistance. A broadening of our understanding is essential for improving treatment outcomes.

Much of the human genome is transcribed as long non-coding RNAs (lncRNAs), a type of ncRNA $200 \mathrm{nt}$ in length that is not translated, but does influence the regulation of gene expression and chemotherapy resistance in various ways $(7,8)$. For example, lncRNA n375709 expression was significantly increased in paclitaxel-resistant CNE-2 nasopharyngeal carcinoma compared with parental CNE-2 cells (9). Knockdown of AK126698 activated the canonical Wnt signaling pathway and induced cisplatin resistance in A549 human lung cancer cells (10). lncRNA MEG3 overexpression in drug resistant A549/DDP cells increased their chemosensitivity to cisplatin both in vitro and in vivo by inhibiting cell proliferation and inducing apoptosis (11). Upregulation of lncRNA GAS5 overcame resistance of human lung adenocarcinoma cells to epidermal growth factor receptor (EGFR) tyrosine kinase inhibitor (TKI) therapy (12). Other lncRNAs, such as HOTTIP, NEAT1, PVT1 and ODRUL have also been reported to modulate chemosensitivity (13-16). It has thus been established that the upregulation and downregulation of lncRNAs is implicated in chemotherapy resistance.

To the best of our knowledge, there have been no studies concerning the roles of lncRNAs in the acquisition of paclitaxel 
resistance in lung adenocarcinoma. We investigated differential expression of lncRNAs and mRNAs in paclitaxel-sensitive A549 and paclitaxel-resistant A549/PTX cells using microarray assays. Nine differentially expressed lncRNAs and six mRNAs were randomly selected for validation by real-time quantitative PCR (RT-qPCR). Subsequent bioinformatics evaluation by Gene Ontology (GO) and Kyoto Encyclopedia of Genes and Genomes (KEGG) pathway analyses identified classical biological regulatory functions and pathways that were differentially expressed in these cell lines. Our results add to the knowledge concerning the involvement of lncRNAs in the resistance to paclitaxel-based chemotherapy in human lung adenocarcinoma cells, and thus, may provide novel molecular therapeutic targets.

\section{Materials and methods}

Cell lines and cell culture. The A549 human lung adenocarcinoma cell line was obtained from the Cell Bank of the Shanghai Branch of the Chinese Academy of Sciences. A549/PTX cells were established in our laboratory in a stepwise manner by exposing drug-sensitive A549 cells to increasing doses of paclitaxel (PTX; Bristol-Myers Squibb, New York, NY, USA). The cells were cultured in RPMI-1640 medium supplemented with $10 \%$ fetal bovine serum (FBS) and $1 \%$ penicillin-streptomycin (both from Gibco, Carlsbad, CA, USA) at $37^{\circ} \mathrm{C}$ in humidified incubator with $5 \% \mathrm{CO}_{2}$. The A549/PTX cell culture medium also contained $200 \mathrm{ng} / \mathrm{ml}$ PTX to maintain the drug-resistant phenotype. Cells in the logarithmic phase of growth were used in all experimental procedures.

In vitro drug sensitivity assay. To evaluate differences in chemoresistance, cells were seeded into 96 -well plates at $5 \times 10^{3}$ cells/well and incubated with various concentrations of PTX for $48 \mathrm{~h}$. At $48 \mathrm{~h}$, the number of viable cells was assayed with Cell Counting Kit-8 (CCK-8; Dojindo Laboratories, Kumamoto, Japan). CCK- 8 reagent (10 $\mu \mathrm{l})$ was added to each well and incubation followed for $1 \mathrm{~h}$ at $37^{\circ} \mathrm{C}$. The number of viable cells was estimated by assessment of the optical density (OD) at $450 \mathrm{~nm}$, and the PTX concentration that produced $50 \%$ inhibition of growth $\left(\mathrm{IC}_{50}\right)$ was estimated from the relative survival curves. Three independent experiments were performed in five duplicate wells.

RNA extraction and RNA quality control. Total RNA was extracted using TRIzol reagent (Takara, Otsu, Japan) following the manufacturer's protocol. RNA quantity and quality were assessed using a NanoDrop ND-1000 spectrophotometer (Thermo Fisher Scientific, Inc., Waltham, MA, USA), and RNA integrity was assessed by electrophoresis on a denaturing agarose gel. Isolated RNAs were stored at $-80^{\circ} \mathrm{C}$ prior to lncRNA microarray analysis and RT-qPCR.

lncRNA microarray. The GeneChip Human Transcriptome Array 2.0 (Affymetrix, Santa Clara, CA, USA) contains $>6.0$ million distinct probes of both coding and non-coding transcripts. Approximately 25,000 lncRNAs and 24,500 mRNAs listed in databanks including RefSeq, Ensembl, UCSC (known genes and lincRNA transcripts), NONCODE, the Human Body Map lincRNA and TUCP catalog, and lncRNAdb, were detected.
RNA labeling and microarray hybridization. RNA labeling and microarray hybridization were performed following an Affymetrix GeneChip protocol from GMINIX BioTech (Shanghai, China). Briefly, total RNA was purified after removal of rRNA and tRNA. Each sample was amplified and transcribed into fluorescent cRNA along the entire length of the transcript, without the 3 ' bias, using a random priming method. After purification, the labeled cRNAs were hybridized at $45^{\circ} \mathrm{C}$ for $16 \mathrm{~h}$ in an Affymetrix hybridization oven. After being washed, the hybridized arrays were scanned using Affymetrix GeneChip Scanner 3000, and data were extracted using Transcriptome Analysis Console Software.

Validation of aberrantly expressed lncRNAs and mRNAs by $R T-q P C R$. Total RNA was extracted using TRIzol reagent, and was then reverse-transcribed using a PrimeScript RT reagent kit with gDNA Eraser (Perfect Real Time; Takara) following the manufacturer's instructions. Reactants were incubated for $2 \mathrm{~min}$ at $42^{\circ} \mathrm{C}, 15 \mathrm{~min}$ at $37^{\circ} \mathrm{C}, 5 \mathrm{sec}$ at $85^{\circ} \mathrm{C}, 7 \mathrm{~min}$ at $4^{\circ} \mathrm{C}$, and then stored at $-20^{\circ} \mathrm{C}$. The transcripts of nine differentially expressed lncRNAs and six mRNAs were randomly selected for RT-qPCR using a SYBR-Green assay (Takara, Dalian, China); GAPDH was used as an internal control. Specific lncRNA and mRNA primers were designed using Primer 5.0. The RT-qPCR reaction was set at an initial denaturation step of $10 \mathrm{~min}$ at $95^{\circ} \mathrm{C}$ followed by 40 cycles of $95^{\circ} \mathrm{C}$ for $10 \mathrm{sec}$ and $60^{\circ} \mathrm{C}$ for $1 \mathrm{~min}$. All experiments were performed three times, and fold changes of expression were calculated using the $2^{-\Delta \Delta \mathrm{Ct}}$ method.

Bioinformatics analysis. Affymetrix Transcriptome Analysis Console software was used to analyze the acquired array images. Differentially expressed lncRNAs and mRNAs were identified through fold-change filtering. GO categories (www. geneontology.org) and KEGG pathway analyses (http://www. genome.jp/kegg/) were performed using the standard enrichment computation method.

Statistical analysis. Statistical analysis was performed using SPSS version 17.0 software (SPSS, Inc., Chicago, IL, USA). Results are presented as the means \pm standard deviation (SD) of three separate assays. Differences between groups were assessed by two tailed t-tests. $\mathrm{P}<0.05$ was considered as statistically significant.

\section{Results}

CCK-8 assay of in vitro drug sensitivity. The paclitaxel-resistance of A549/PTX cells was characterized by determining the $\mathrm{IC}_{50}$ value. After treatment with various concentrations of paclitaxel for $48 \mathrm{~h}$ in A549 and A549/PTX cells, the cell survival was assayed using CCK-8 (Fig. 1A). The paclitaxel $\mathrm{IC}_{50}$ value for the drug-resistant A549/PTX cell line was 1240.12 \pm 6.13 and $18.21 \pm 0.84 \mathrm{ng} / \mathrm{ml}$ for the A549 cell line (Fig. 1B), with a drug-resistance index of A549/PTX relative to A549 cells of $68(\mathrm{P}<0.01)$. A previously reported criterion of high-resistance is an index $>20$ (17). The A549/PTX cells were revealed to be more resistant to paclitaxel than the A549 cells.

RNA quality control. RNA quantification and quality were spectrophotometrically assayed using the NanoDrop ND-1000. 

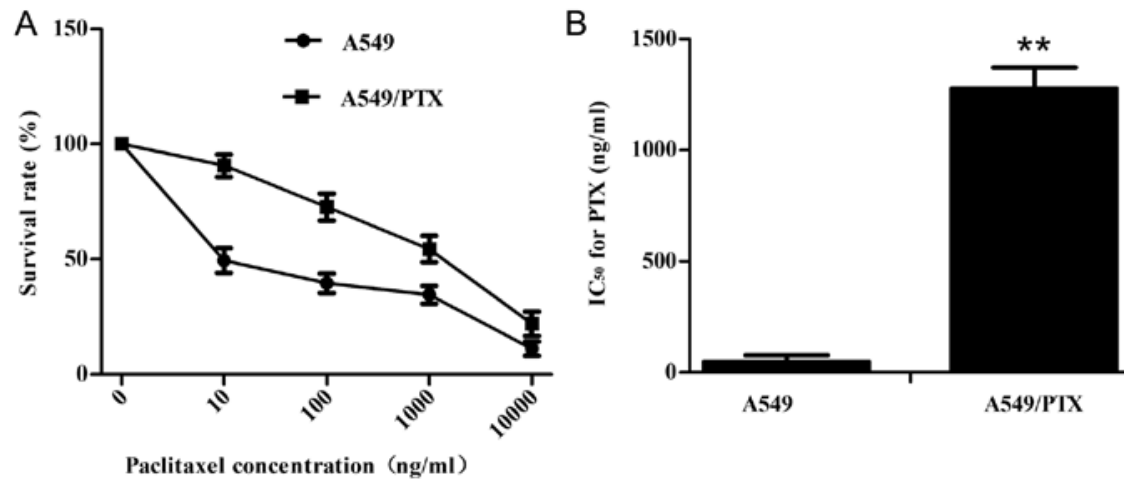

Figure 1. Paclitaxel (PTX)-resistance of A549 and A549/PTX cells. (A) Cells were treated with increasing concentrations of paclitaxel and $48 \mathrm{~h}$ later, the cell survival rate was assessed using CCK- 8 assay. The A549/PTX cells were more resistant to paclitaxel than the A549 cells. (B) The IC $_{50}$ value of paclitaxel for the A549/PTX cells was $1240.12 \pm 6.13 \mathrm{ng} / \mathrm{ml}$, and for the A549 cells the value was $18.21 \pm 0.84 \mathrm{ng} / \mathrm{ml}$. The drug-resistance index of A549/PTX cells relative to A549 cells was 68 . Data are presented as the mean \pm standard deviation; ${ }^{* *} \mathrm{P}<0.01$.

A

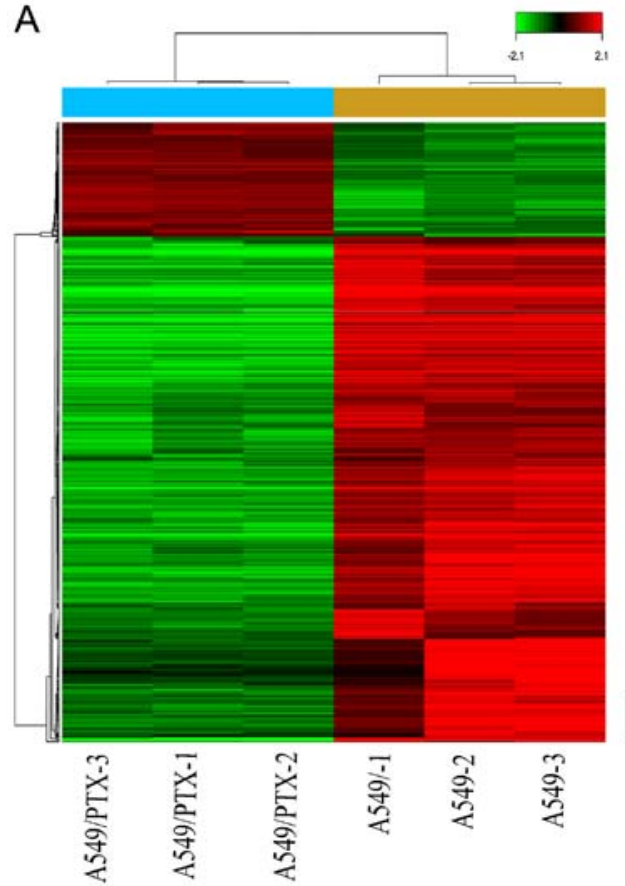

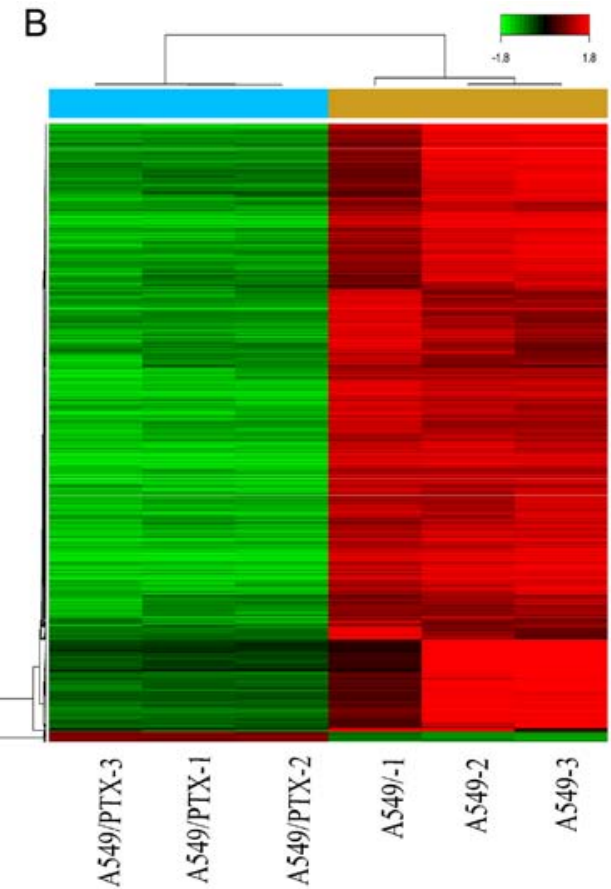

Figure 2. Microarray expression profiling of differentially expressed long non-coding RNAs (lncRNAs) and mRNAs in A549 and paclitaxel-resistant A549 (A549/PTX) cells. (A) Hierarchical clustering of all target lncRNAs. (B) Hierarchical clustering of all target mRNAs. Each row represents one lncRNA or mRNA, and each column represents one cell line sample. 'Red' indicates high relative expression, and 'green' indicates low relative expression. There were 1,154 lncRNAs and 1,733 mRNAs that exhibited a >3-fold differential expression in A549/PTX cells compared with A549 cells. Among them, more were downregulated.

The OD A260/A280 ratio of the total RNA was between 1.8 and 2.1. RNA integrity was assessed by electrophoresis on a denaturing agarose gel. The $28 \mathrm{~s}$ and $18 \mathrm{~s}$ ribosomal RNA bands were fairly sharp and intense, and the 28s rRNA band was about twice as intense as the 18s rRNA band. The lncRNA and mRNA samples from the A549 and A549/PTX cells were found to be suitable for microarray expression profiling.

Differentially expressed lncRNAs and mRNAs. The Affymetrix GeneChip Human Transcriptome Array 2.0 was used for profiling both parental A549 and A549/PTX cells. For microarray analysis, 25,000 lncRNAs and 24,500 mRNAs were detected. After quantile normalization and data filtering, we found a $>3$-fold difference in the expression of
1,154 lncRNAs and 1,733 mRNAs in the A549/PTX cells compared with the A549 cells. Of the 1,154 differentially expressed lncRNAs, 119 were upregulated and 1,035 were downregulated $(\mathrm{P}<0.01) ; 28$ of the mRNAs were upregulated and 1,705 were downregulated $(\mathrm{P}<0.01)$. The hierarchical cluster analysis of the expression of the lncRNAs and mRNAs that were identified is shown in Fig. 2. The differentially expressed lncRNAs and mRNAs are listed in Table I, and the 10 lncRNAs and mRNAs with the greatest relative upregulation or downregulation are shown in Tables II and III. lncRNA n334075 (fold change $=8.77$ ) was the most significantly upregulated and lncRNA n335556 (fold change $=-308.61$ ) was the most significantly downregulated. Norrin cystine knot growth factor (NDP) mRNA (fold change $=6.75$ ) was the most 
Table I. Number of differentially expressed IncRNAs and mRNAs.

\begin{tabular}{lcc}
\hline lncRNAs and mRNAs & Fold change $>3$ & Total \\
\hline lncRNAs & & 1,154 \\
$\quad$ Upregulation & 119 & \\
Downregulation & 1,035 & \\
mRNAs & & 1,733 \\
Upregulation & 28 & \\
Downregulation & 1,705 & \\
\hline
\end{tabular}

lncRNAs, long non-coding RNAs.

Table II. Ten most significantly upregulated and downregulated lncRNAs.

\begin{tabular}{|c|c|c|c|}
\hline \multicolumn{2}{|c|}{ Upregulated in A549/PTX } & \multicolumn{2}{|c|}{ Downregulated in A549/PTX } \\
\hline lncRNAs & $\begin{array}{l}\text { Fold } \\
\text { change }\end{array}$ & lncRNAs & $\begin{array}{c}\text { Fold } \\
\text { change }\end{array}$ \\
\hline n334075 & 8.77 & n335556 & -308.61 \\
\hline n333225 & 8.76 & n333732 & -191.67 \\
\hline n335243 & 8.04 & n385730 & -154.98 \\
\hline ENST00000455973 & 7.86 & n342204 & -120.84 \\
\hline ENST00000416226 & 7.77 & ENST00000544920 & -110.94 \\
\hline NR_045483 & 7.66 & NR_002206 & -95.60 \\
\hline ENST00000486726 & 7.62 & ENST00000363046 & -86.91 \\
\hline ENST00000503218 & 6.98 & NR_002569 & -82.88 \\
\hline n335614 & 5.86 & NR_002555 & -79.82 \\
\hline ENST00000439079 & 5.21 & NR_029396 & -76.72 \\
\hline
\end{tabular}

A549/PTX vs. parental A549 cells. IncRNAs, long non-coding RNAs; A549/PTX, paclitaxel-resistant A549 cells.

significantly upregulated and histone cluster $2 \mathrm{H} 2 \mathrm{~A}$ family member a4 (HIST2H2AA4) (fold change=-99.71) was the most significantly downregulated. Downregulated lncRNAs and mRNAs were more common than upregulated lncRNAs and mRNAs in our microarray data.

$R T-q P C R$ validation of microarray data. To validate the microarray data, nine differentially expressed lncRNAs and six mRNAs were randomly selected for RT-qPCR assay of RNA isolated from both resistant A549/PTX and sensitive A549 cells. The primers used for PCR validation are listed in Table IV. As shown in Fig. 3, the expression of lncRNA ENST00000455973,ENST00000416226,ENST00000486726 and ENST00000503218 was upregulated and the expression of ENST00000544920, NR-002206, ENST00000363046, NR-002555 and ENST00000500843 was downregulated. The expression of DDR2 and TNF mRNA was upregulated and the expression of ABCC2, MRPS30, NEDD4, and CASP2 was downregulated. The RT-qPCR results were thus, consistent with the microarray data.
Table III. Ten most significantly upregulated and downregulated mRNAs.

\begin{tabular}{lcllc}
\hline \multirow{2}{*}{ Upregulated in } & A549/PTX & & \multicolumn{2}{l}{ Downregulated in A549/PTX } \\
\cline { 1 - 2 } \cline { 5 - 5 } mRNAs & Fold change & & mRNAs & Fold change \\
\hline NDP & 6.75 & & HIST2H2AA4 & -99.71 \\
TNF & 6.12 & & ALDH1A1 & -92.66 \\
DDR2 & 5.76 & & FTL & -77.03 \\
WNT6 & 5.69 & & PTPLAD1 & -76.91 \\
NCAM1 & 4.58 & & CYP24A1 & -73.79 \\
LOXL1 & 4.47 & & AKR1C2 & -58.71 \\
KRTAP5-6 & 3.42 & & NQO1 & -55.83 \\
IGLV7-43 & 3.39 & & NETO2 & -46.82 \\
IGHV3-48 & 3.38 & & RSL1D1 & -46.32 \\
SPPL2C & 3.34 & & HIST1H1E & -41.36 \\
\hline
\end{tabular}

A549/PTX vs. parental A549 cells. A549/PTX, paclitaxel-resistant A549 cells.

Chromosomal distribution of differentially expressed lncRNAs and mRNAs. Since chromosomal imbalances have been associated with drug resistance, a distribution plot of chromosomal location was developed to show the chromosomal locations of the differentially expressed lncRNAs and mRNAs. As shown in Fig. 4, the 1,154 lncRNAs and 1,733 mRNAs were distributed throughout the genome, and were associated with every chromosome. In the present study, aberrantly expressed lncRNAs and mRNAs were most frequently found on chromosomes 1, 2,6,12 and 17, however, particularly on chromosome 1 .

GO and KEGG pathway analyses. GO analysis was applied to identify the functions of the differentially expressed mRNAs. The GO database includes the primary functional classifications of the National Center for Biotechnology Information (NCBI), and includes three categories, i.e., cellular components, molecular functions and biological processes. The microarray data obtained in the present study revealed that the differentially expressed genes were enriched for GO terms related to the cytosol, cytoplasm, nucleus and nucleolus (cellular components); protein, ATP and RNA bindings, and structural constituents of ribosomes (molecular functions); and gene expression, mitotic cell cycle, small-molecule metabolic process, and translation (biological processes). The top $10 \mathrm{GO}$ terms within each of the three categories, cellular components, molecular functions and biological processes, are shown in Fig. 5A-C.

We conducted pathway analysis of the differentially expressed mRNAs using the latest KEGG database, which identified the biological pathways involved in paclitaxel resistance. We found 96 pathways corresponding to aberrant mRNAs, and the predominant pathways are shown in Fig. 5D. The top 10 pathways were metabolic, ribosomal, RNA transport, cell cycle, endocytosis, pathways in cancer, oxidative phosphorylation, purine metabolism, mismatch repair and DNA replication. 
Table IV. Primers used for RT-qPCR validation.

\begin{tabular}{lll}
\hline IncRNAs and mRNAs & \multicolumn{1}{c}{ Forward primer sequence } & Reverse primer sequence \\
\hline ENST00000363046 & GGACTCTGTTCCTCCCCTTTC & GAGCCCCGTGTGGTTGG \\
ENST00000500843 & CCTGGCTGAGGTGAATAA & TTGGACCCGAACATCTG \\
ENST00000544920 & AAAGATGAGGCAGAGGTCCAAG & CGATCAGAGGGCGATGAAG \\
NR-002555 (LOC613037) & GGGCAGAGGACTACCACAAATG & TGTTGTTGAGTTGGAGGAGGTG \\
NR-002206 (GTF2IP1) & GCTGTGTGGTGGTTGATGG & CTCTTTTATTTCTTCTGTGGCTGGA \\
ENST00000455973 & GATGTGGGAAACAGTGGC & GTAAGGCAGCAGGGAGG \\
ENST00000416226 & AGGAGAAACTCATCAGGC & ATCTCTTCTACGGTGGCT \\
ENST00000486726 & CCTGTCTGGTGTCCTTGC & CAGCAGGAGAGGCATCAG \\
ENST00000503218 & GCAAGTGAAGCCTGATACC & AAAGCGTCTGTGAGCCTAA \\
TNF & GTGACAAGCCTGTAGCCCATGTT & TTATCTCTCAGCTCCACGCCATT \\
DDR2 & CCCAGCTGTCAGATGAACAGGTTA & TCAGGACAAATGGCTGGTTGAG \\
CASP2 & TGGCATGCATCCTCATCATC & TCTGGCTGAAACTGCCCACT \\
ABCC2 & AGTGATCACCATCGCCCACA & GTTCACATTCTCAATGCCAGCTTC \\
MRPS30 & CGAACCCGAACCTGAACCT & GATATGACCTCGCTCTCCTCGT \\
NEDD4 & TGAAGCCCAATGGGTCAGAAATA & GGACCCTGTTCACAAATCTCCAC \\
GAP & TGCACCACCAACTGCTTAGC & GGCATGGACTGTGGTCATGAG
\end{tabular}
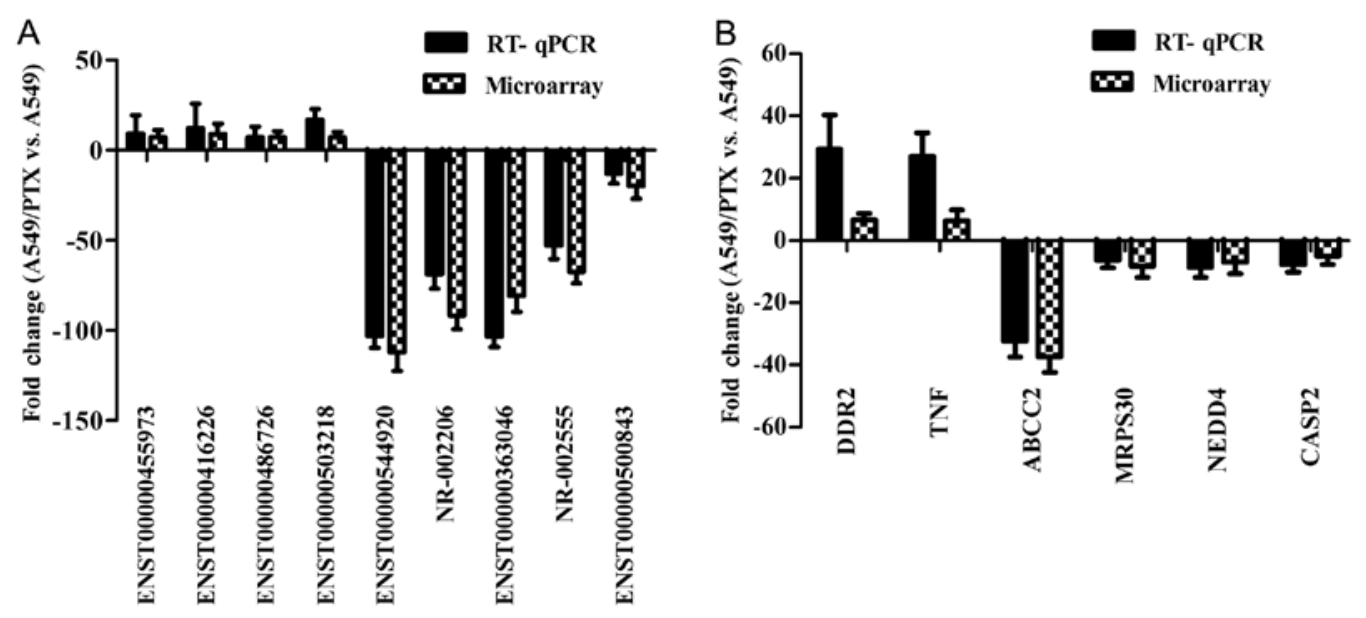

Figure 3. Validation of microarray data by RT-qPCR. (A) Nine long non-coding RNAs (lncRNAs) and (B) six mRNAs differentially expressed in A549 cells compared with paclitaxel-resistant A549 (A549/PTX) cells by microarray were randomly selected and validated by RT-qPCR. The heights of the columns in the chart represent the mean fold change of the expression. The fold change was positive when the expression was upregulated (A549/PTX vs. A549 cells) and negative when the expression was downregulated. Data are presented as the mean \pm standard deviation.

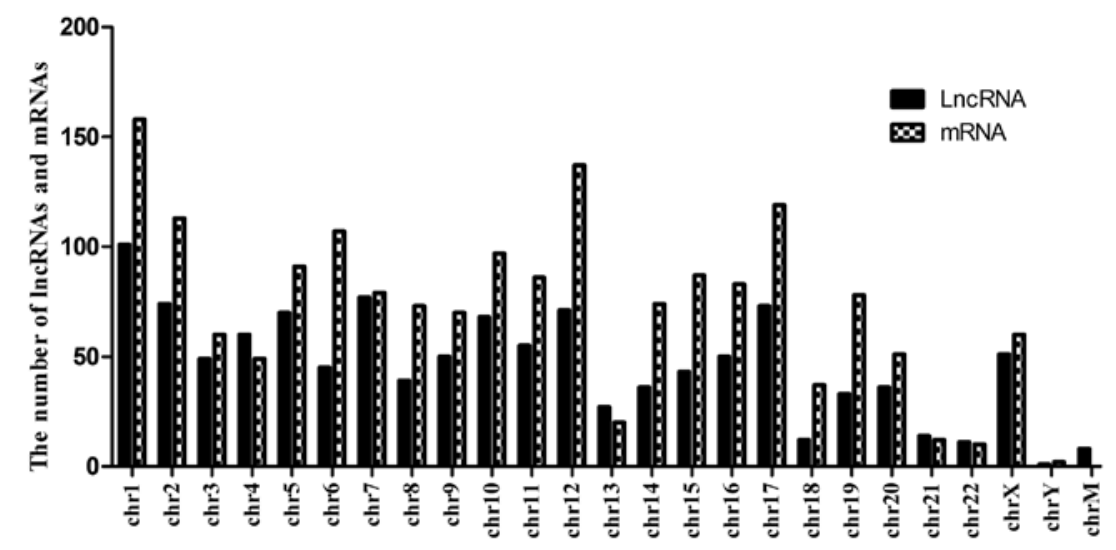

Figure 4. Chromosomal distribution of the differentially expressed long non-coding RNAs (lncRNAs) and mRNAs. The y-axis indicates the number of upregulated and downregulated lncRNAs and mRNAs. Aberrantly expressed lncRNAs and mRNAs were found to be mainly located on chromosomes 1, 2, 6, 12 and 17. 'chr', chromosome. 
A

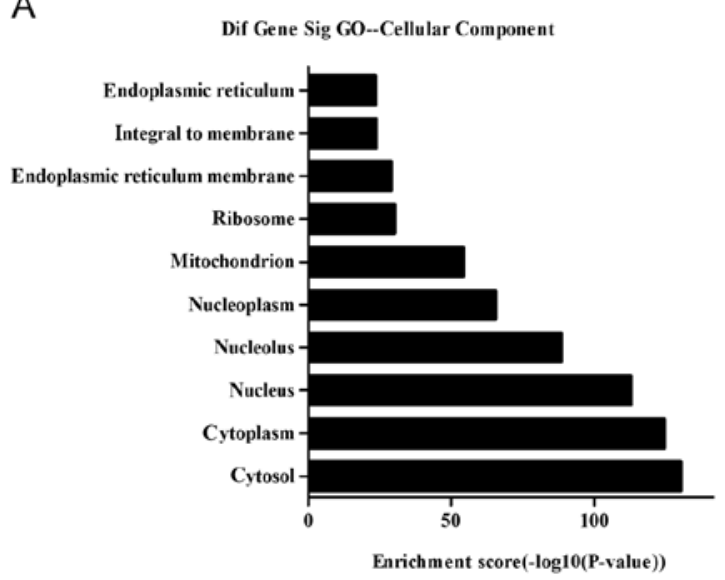

C

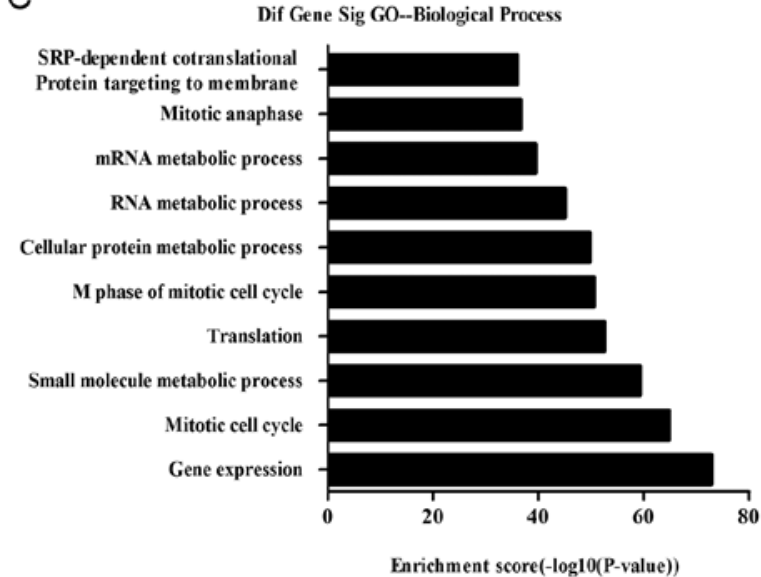

B

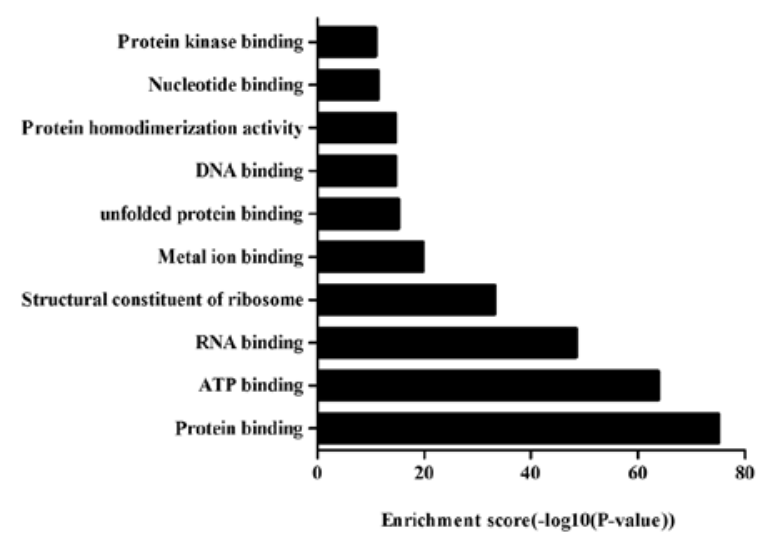

D

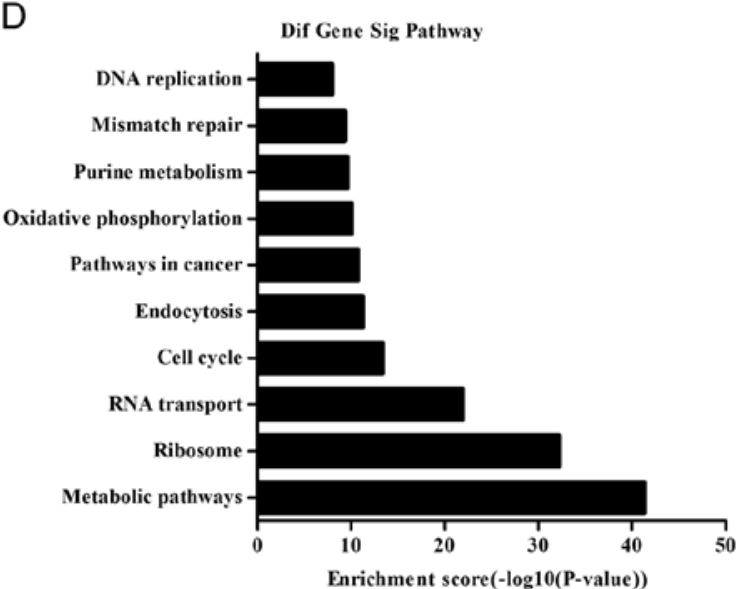

Figure 5. Analysis of significant GO and Kyoto Encyclopedia of Gene and Genome (KEGG) pathways. (A-C) GO and (D) KEGG pathways analyses. The histograms show the top 10 significant GO and KEGG pathways of the differentially expressed genes. The P-value (Fisher P-value) denotes the significance of the GO and the KEGG pathway enrichment. The lower the P-value, the more significant was the difference in the GO and KEGG pathways (the recommend P-value cut-off is 0.05).

\section{Discussion}

Paclitaxel is the standard and most effective chemotherapy drug used for lung adenocarcinoma, but drug resistance is a major clinical obstacle that limits its clinical benefits (18). Although some molecular mechanisms of paclitaxel resistance in various types of cancers have been reported in the past few decades (19), the precise cause of paclitaxel resistance in lung adenocarcinoma remains unclear. Research is urgently warranted to fully understand and overcome it. Evidence that IncRNAs are involved in chemotherapy resistance is increasing (20-22), but to the best of our knowledge there is little data on the correlations between IncRNAs and paclitaxel resistance in human lung adenocarcinoma.

To investigate the regulatory effects of lncRNAs in paclitaxel resistance of lung adenocarcinoma, we established a paclitaxel-resistant A549/PTX cell line and assayed its PTX resistance. Microarray expression profiling of lncRNAs and mRNAs in parental A549 and paclitaxel resistant A549/PTX cells identified a total of 1,154 differentially expressed lncRNAs. We also found aberrant expression of 1,733 mRNAs in A549/PTX cells compared with the parental A549 cells. Various of the differentially expressed lncRNAs and mRNAs including, MALAT1, TUG1, HOTAIR, ABCB1
(MDR1) and Wnt6 have been previously reported in other chemoresistant cancers (23-27). Whether the molecular mechanisms of chemoresistance are the same as in the A549/PTX cells warrants further investigation. However, in our screening results, the 10 predominantly upregulated and downregulated IncRNAs have not been previously related to treatment resistance. Consequently, their mechanism of resistance regulation and their function in resistant cells are not clear. lncRNAs regulate neighboring protein-coding genes (28), and we found that some of the aberrantly expressed lncRNAs may play important roles in paclitaxel resistance by regulating nearby coding genes. For example, upregulated lncRNA ENST00000447028 was found to be located near VEGF-B mRNA. VEGF-B mRNA was reported by Yang et al to take part in the resistance of lung cancer cells to the chemotherapeutic drug EGFR-TKI (29). We found that VEGF-B mRNA was strongly upregulated, therefore, ENST00000447028 may influence paclitaxel resistance by regulating VEGF-B mRNA. Another upregulated IncRNA, ENST00000416226, was found to be located near Wnt6 mRNA, which was upregulated in our results, and is involved in both oncogenesis and chemoresistance in human cancers (30). We thus, speculate that IncRNAs may influence paclitaxel resistance in human lung adenocarcinoma by regulating the expression of their nearby 
coding genes. Further studies of the effect of overexpression or knockdown of lncRNAs and western blot analyses should be performed to investigate the precise relationships.

We found that the aberrantly expressed lncRNAs and mRNAs were distributed throughout the genome and could be found in every chromosome, particularly on chromosome 1. Perhaps, all the chromosomes participate in paclitaxel resistance. The importance of chromosome 1 in the occurrence of paclitaxel resistance in lung adenocarcinoma warrants investigation.

The biological functions and signaling pathways associated with the lncRNAs and mRNAs identified in the present study, were evaluated by GO and KEGG pathway analyses. The most enriched GOs were cytosol (cellular components), protein binding (molecular function) and gene expression (biological processes). In addition, many of the identified GO terms in our results have been reported in other cancer chemoresistance studies. For example, protein and nucleotide binding, and metabolic processes have been reported to be involved in the chemoresistance of lung and colorectal cancer (31). This suggests that lncRNAs among those we identified may have regulated chemoresistance of the A549/PTX cells by influencing the expression of these GO database genes. Pathway analysis revealed a total of 96 pathways corresponding to all the differentially expressed mRNAs. Some of the top 10 pathways that we identified have previously been associated with chemoresistance. For example, Wu et al using genome-wide microarrays found that the metabolic pathway participated in EGFR-TKI resistance of lung adenocarcinoma (32). Zhu et al reported that the cell cycle pathway was associated with the development of doxorubicin resistance in the MG63/DXR human osteosarcoma cell line (33). Zhou et al found that the DNA replication pathway contributed to the occurrence of gemcitabine resistance in SW1990 pancreatic cancer cells (34). These data revealed that the differentially expressed lncRNAs may regulate paclitaxel resistance in lung adenocarcinoma through these classical pathways.

In conclusion, the present study reveals, for the first time, numerous differentially expressed lncRNAs and mRNAs in A549 and A549/PTX cells which may play an important role in regulating paclitaxel resistance through various biological functions and signaling pathways. We described a novel approach to clarify the molecular mechanisms of paclitaxel resistance in human lung adenocarcinoma. The results provide a novel rationale for studies on reversal of paclitaxel resistance and for identifying patients who can benefit the most from chemotherapy. Evaluation of clinical samples for in vivo validation is warranted.

\section{Acknowledgements}

The present study was supported by the National Natural Science Foundation of China (grant no. 31571198), and the Outstanding Scientific Fund of Shengjing Hospital (no. 201601).

\section{References}

1. Jemal A, Bray F, Center MM, Ferlay J, Ward E and Forman D: Global cancer statistics. CA Cancer J Clin 61: 69-90, 2011.
2. Morgensztern D, Ng SH, Gao F and Govindan R: Trends in stage distribution for patients with non-small cell lung cancer: A National Cancer Database survey. J Thorac Oncol 5: 29-33, 2010.

3. Sun QL, Sha HF, Yang XH, Bao GL, Lu J and Xie YY: Comparative proteomic analysis of paclitaxel sensitive A549 lung adenocarcinoma cell line and its resistant counterpart A549-Taxol. J Cancer Res Clin Oncol 137: 521-532, 2011.

4. Khongkow P, Gomes AR, Gong C, Man EP, Tsang JW, Zhao F, Monteiro LJ, Coombes RC, Medema RH, Khoo US, et al: Paclitaxel targets FOXM1 to regulate KIF20A in mitotic catastrophe and breast cancer paclitaxel resistance. Oncogene 35: 990-1002, 2016.

5. Park SH, Seong MA and Lee HY: p38 MAPK-induced MDM2 degradation confers paclitaxel resistance through p53-mediated regulation of EGFR in human lung cancer cells. Oncotarget 7: 8184-8199, 2016.

6. Holleman A, Chung I, Olsen RR, Kwak B, Mizokami A, Saijo N, Parissenti A, Duan Z, Voest EE and Zetter BR: miR-135a contributes to paclitaxel resistance in tumor cells both in vitro and in vivo. Oncogene 30: 4386-4398, 2011.

7. Hirano T, Yoshikawa R, Harada H, Harada Y, Ishida A and Yamazaki T: Long noncoding RNA, CCDC26, controls myeloid leukemia cell growth through regulation of KIT expression. Mol Cancer 14: 90, 2015.

8. Wang Y, Wu K, Yang Z, Zhao Q, Fan D, Xu P, Nie Y and Fan D: Multidrug-resistance related long non-coding RNA expression profile analysis of gastric cancer. PLoS One 10: e0135461, 2015.

9. Ren S, Li G, Liu C, Cai T, Su Z, Wei M, She L, Tian Y, Qiu Y, Zhang $\mathrm{X}$, et al: Next generation deep sequencing identified a novel lncRNA n375709 associated with paclitaxel resistance in nasopharyngeal carcinoma. Oncol Rep 36: 1861-1867, 2016.

10. Yang Y, Li H, Hou S, Hu B, Liu J and Wang J: The noncoding RNA expression profile and the effect of lncRNA AK126698 on cisplatin resistance in non-small-cell lung cancer cell. PLoS One 8: e65309, 2013.

11. Liu J, Wan L, Lu K, Sun M, Pan X, Zhang P, Lu B, Liu G and Wang Z: The long noncoding RNA MEG3 contributes to cisplatin resistance of human lung adenocarcinoma. PLoS One 10: e0114586, 2015.

12. Dong S, Qu X, Li W, Zhong X, Li P, Yang S, Chen X, Shao M and Zhang L: The long non-coding RNA, GAS5, enhances gefitinib-induced cell death in innate EGFR tyrosine kinase inhibitor-resistant lung adenocarcinoma cells with wide-type EGFR via downregulation of the IGF-1R expression. J Hematol Oncol 8: 43, 2015.

13. Li Z, Zhao X, Zhou Y, Liu Y, Zhou Q, Ye H, Wang Y, Zeng J, Song Y, Gao W, et al: The long non-coding RNA HOTTIP promotes progression and gemcitabine resistance by regulating HOXA13 in pancreatic cancer. J Transl Med 13: 84, 2015.

14. Gao C, Zhang J, Wang Q and Ren C: Overexpression of lncRNA NEAT1 mitigates multidrug resistance by inhibiting ABCG2 in leukemia. Oncol Lett 12: 1051-1057, 2016.

15. Ding J, Li D, Gong M, Wang J, Huang X, Wu T and Wang C: Expression and clinical significance of the long non-coding RNA $P V T 1$ in human gastric cancer. Onco Targets Ther 7: 1625-1630, 2014.

16. Zhang CL, Zhu KP, Shen GQ and Zhu ZS: A long non-coding RNA contributes to doxorubicin resistance of osteosarcoma. Tumour Biol 37: 2737-2748, 2016.

17. Snow K and Judd W: Characterisation of adriamycin- and amsacrine-resistant human leukaemic $\mathrm{T}$ cell lines. Br J Cancer 63: 17-28, 1991.

18. Liu R, Liu X, Zheng Y, Gu J, Xiong S, Jiang P, Jiang X, Huang E, Yang Y, Ge D, et al: MicroRNA-7 sensitizes non-small cell lung cancer cells to paclitaxel. Oncol Lett 8: 2193-2200, 2014.

19. Chatterjee A, Chattopadhyay D and Chakrabarti G: miR-17-5p downregulation contributes to paclitaxel resistance of lung cancer cells through altering beclin1 expression. PLoS One 9: e95716, 2014.

20. Lee H, Kim C, Ku JL, Kim W, Yoon SK, Kuh HJ, Lee JH, Nam SW and Lee EK: A long non-coding RNA snaR contributes to 5-fluorouracil resistance in human colon cancer cells. Mol Cells 37: 540-546, 2014

21. You L, Chang D, Du HZ and Zhao YP: Genome-wide screen identifies PVT1 as a regulator of Gemcitabine sensitivity in human pancreatic cancer cells. Biochem Biophys Res Commun 407: 1-6, 2011.

22. Fang S, Gao H, Tong Y, Yang J, Tang R, Niu Y, Li M and Guo L: Long noncoding RNA-HOTAIR affects chemoresistance by regulating HOXA 1 methylation in small cell lung cancer cells. Lab Invest 96: 60-68, 2016. 
23. Jiao F, Hu H, Han T, Yuan C, Wang L, Jin Z, Guo Z and Wang L: Long noncoding RNA MALAT-1 enhances stem cell-like phenotypes in pancreatic cancer cells. Int J Mol Sci 16: 6677-6693, 2015.

24. Li J, Zhang M, An G and Ma Q: LncRNA TUG1 acts as a tumor suppressor in human glioma by promoting cell apoptosis. EExp Biol Med 241: 644-649, 2016.

25. Liu Z, Sun M, Lu K, Liu J, Zhang M, Wu W, De W, Wang Z and Wang R: The long noncoding RNA HOTAIR contributes to cisplatin resistance of human lung adenocarcinoma cells via downregualtion of $\mathrm{p} 21^{\mathrm{WAF} / \mathrm{CIP} 1}$ expression. PLoS One 8: e77293, 2013.

26. Aldonza MB, Hong JY, Bae SY, Song J, Kim WK, Oh J, Shin Y, Lee SH and Lee SK: Correction: Suppression of MAPK signaling and reversal of mTOR-dependent MDR1-associated multidrug resistance by $21 \alpha$-methylmelianodiol in lung cancer cells. PLoS One 10: e0127841, 2015.

27. Fan Y, Shen B, Tan M, Mu X, Qin Y, Zhang F and Liu Y: Long non-coding RNA UCA1 increases chemoresistance of bladder cancer cells by regulating Wnt signaling. FEBS J 281: 1750-1758, 2014.

28. Cheng N, Li X, Zhao C, Ren S, Chen X, Cai W, Zhao M, Zhang Y, Li J, Wang Q, et al: Microarray expression profile of long noncoding RNAs in EGFR-TKIs resistance of human non-small cell lung cancer. Oncol Rep 33: 833-839, 2015.

29. Yang X, Zhang Y, Hosaka K, Andersson P, Wang J, Tholander F, Cao Z, Morikawa H, Tegnér J, Yang Y, et al: VEGF-B promotes cancer metastasis through a VEGF-A-independent mechanism and serves as a marker of poor prognosis for cancer patients. Proc Natl Acad Sci USA 112: E2900-E2909, 2015.
30. Su HY, Lai HC, Lin YW, Liu CY, Chen CK, Chou YC, Lin SP, Lin WC, Lee HY and Yu MH: Epigenetic silencing of SFRP5 is related to malignant phenotype and chemoresistance of ovarian cancer through Wnt signaling pathway. Int J Cancer 127: 555-567, 2010.

31. Xiong W, Jiang YX, Ai YQ, Liu S, Wu XR, Cui JG, Qin JY, Liu Y, $\mathrm{Xia} \mathrm{YX}, \mathrm{Ju} \mathrm{YH}$, et al: Microarray analysis of long non-coding RNA expression profile associated with 5-fluorouracil-based chemoradiation resistance in colorectal cancer cells. Asian Pac J Cancer Prev 16: 3395-3402, 2015.

32. Wu Y, Yu DD, Hu Y, Yan D, Chen X, Cao HX, Yu SR, Wang Z and Feng JF: Genome-wide profiling of long non-coding RNA expression patterns in the EGFR-TKI resistance of lung adenocarcinoma by microarray. Oncol Rep 35: 3371-3386, 2016.

33. Zhu KP, Zhang CL, Shen GQ and Zhu ZS: Long noncoding RNA expression profiles of the doxorubicin-resistant human osteosarcoma cell line MG63/DXR and its parental cell line MG63 as ascertained by microarray analysis. Int J Clin Exp Pathol 8: 8754-8773, 2015.

34. Zhou M, Ye Z, Gu Y, Tian B, Wu B and Li J: Genomic analysis of drug resistant pancreatic cancer cell line by combining long non-coding RNA and mRNA expression profling. Int J Clin Exp Pathol 8: 38-52, 2015. 